\title{
CONSULTA DE ENFERMAGEM PRÉ-TRANSPLANTE RENAL: UMA ATIVIDADE RELACIONADA AO AUTOCUIDADO
}

\author{
Nursing Consultation Before kidney transplantation: an activity related to the self-care
}

Maria Conceição da Costa Proença ${ }^{1}$, Alessandra Rosa Vicari ${ }^{1}$, Débora Hexsel Gonçalves ${ }^{1,2}$

\begin{abstract}
RESUMO
A consulta de enfermagem é um recurso utilizado pelo enfermeiro, no cuidado ao paciente, família, grupos ou comunidades, priorizando a autonomia e a independência do indivíduo nos aspectos preventivos e educativos, através do planejamento da assistência, da verificação dos fatores de risco, da determinação dos diagnósticos de enfermagem e da implementação das intervenções, buscando resultados. O objetivo deste trabalho é relatar a experiência da consulta de enfermagem pré-transplante renal como atividade educativa para pacientes que ingressam em lista de espera. Trata-se de um estudo descritivo, qualitativo e baseado no relato de experiência na realização de consultas de enfermagem com pacientes que ingressam em lista de espera para transplante renal. Utiliza-se um modelo informatizado de anamnese e exame físico e Evolução de Enfermagem, o qual está estruturado conforme recomendado por Weed (Subjetivo, Objetivo, Análise ou Interpretação e Plano ou Conduta) ${ }^{8}$, utilizando o referencial teórico de Wanda Horta, baseado nas necessidades humanas básicas ${ }^{9}$. Este sistema denominado Aplicativos de Gestão Hospitalar (AGH) foi elaborado pelos profissionais, segundo suas áreas de atuação e pela equipe de analistas do sistema de informatização do HCPA. Como resultados, observamos que a consulta de enfermagem pré-transplante renal com base neste modelo e referencial teórico permite ao enfermeiro avaliar as habilidades do paciente para realizar o autocuidado, realizar um plano de cuidados individualizado, levando em consideração a experiência e o conhecimento do paciente, da família, do grupo ou comunidade e realizar encaminhamentos a outras especialidades como: nutrição, odontologia, ginecologia, assistência social, entre outros.
\end{abstract}

Descritores: Enfermagem, Transplante renal, Ambulatório hospitalar.

\footnotetext{
Instituições:

1 Hospital de Clínicas de Porto Alegre, Porto Alegre, RS, Brasil

2 Escola de Enfermagem da Universidade Federal do Rio Grande do Sul-EEUFRGS, Porto Alegre, RS, Brasil
}

Correspondência:

Maria Conceição da Costa Proença

Rua Dona Laura, 60 / 302 - Porto Alegre / RS - Brasil - CEP 90430-090

Tel.: (51) 3222-9741 / (51) 9971-1462

E-mail:mproenca@hcpa.ufrgs.br

Recebido em: 13.10.2009

\section{INTRODUÇÃO}

A Consulta de Enfermagem está contemplada como atividade privativa do enfermeiro, na lei $^{1}$ do exercício profissional $\mathrm{n}^{\mathrm{o}}$. 7.498/86, no seu art.11, inciso I, e vem sendo efetivada na prática por enfermeiros que acreditam nela como um instrumento sistematizado para assistência de enfermagem.

A Consulta de Enfermagem é um recurso utilizado pelo enfermeiro direcionado a prestar diversas atividades relacionadas ao paciente, família, grupos ou comunidades, priorizando a autonomia e a independência do indivíduo em aspectos preventivos e educativos através do planejamento da assistência, verificação dos fatores de risco, determinação dos Diagnósticos de Enfermagem e implementação das intervenções, buscando resultados. ${ }^{2}$

O processo de educar em saúde, parte essencial do trabalho de cuidar da enfermagem, pode ser entendido como "um diálogo que se trava entre as pessoas com o objetivo de mobilizar forças e a motivação para mudanças, seja de comportamento, atitude ou adaptações às novas situações de vida". ${ }^{3} \mathrm{O}$ profissional da enfermagem deve ser um instrumento para que o cliente adquira autonomia no agir, aumentando a capacidade de enfrentar situações de estresse, de crise e decida sobre a vida e a saúde. ${ }^{4,5}$ 
O enfermeiro, ao realizar a consulta, assume responsabilidades quanto à ação da enfermagem a ser prestada nos problemas detectados e, em nível de complexidade da intervenção: a) cuidados diretos e indiretos necessários; b) orientações indicadas para a situação; c) encaminhamento para outros profissionais (quando a competência de resolução do problema foge de seu âmbito de ação). Portanto, o procedimento requer o desenvolvimento de habilidades para a tomada de decisão, de forma a proporcionar uma assistência integral e eficaz. Essa assistência não deve ser centrada na doença nem no indivíduo hospitalizado, mas em todas as pessoas que, ante seus conflitos e dificuldades, venham mostrar por seu comportamento necessidade de ajuda. ${ }^{6}$

O processo de educar-cuidar ocorre em uma relação horizontal, dialógica, recíproca e verdadeiramente humana. Tanto o paciente quanto a enfermeira (o) são sujeitos que participam de uma relação de cuidado, e que, como seres orgânicos, estão sempre se transformando, imbuídos de pensamentos e ações que se refazem e se modificam ao longo de sua trajetória existencial. ${ }^{7}$

\section{OBJETIVO}

O trabalho tem como objetivo relatar a experiência da Consulta de Enfermagem pré-transplante renal como uma atividade educativa para pacientes que ingressam em lista de espera.

\section{MÉTODOS}

Trata-se de estudo descritivo de caráter qualitativo baseado no relato de experiência de enfermeiras de um hospital universitário de Porto Alegre, Rio Grande do Sul, na realização de Consultas de Enfermagem com pacientes que ingressam em lista de espera para transplante renal. $\mathrm{O}$ instrumento informatizado de Anamnese, Exame físico e Evolução de Enfermagem, denominado Aplicativos de Gestão Hospitalar (AGH) foi elaborado por profissionais das diferentes áreas de atuação e pela equipe de analistas do sistema de informatização do HCPA, o mesmo foi estruturado conforme recomendado por Weed (Subjetivo, Objetivo, Análise ou Interpretação e Plano ou Conduta), utilizando o referencial teórico de Wanda Horta, baseado nas necessidades humanas básicas. ${ }^{8,9}$

\section{RESULTADOS E DISCUSSÃO}

A Consulta de Enfermagem pré-transplante renal permite ao enfermeiro avaliar as habilidades do paciente em realizar autocuidado, seu conhecimento e aderência ao tratamento crônico. Alguns estudos sugerem que a prevalência dos não aderentes é de aproximadamente 54\%, e detectar os pacientes de risco é importante na prevenção da perda do enxerto..$^{10,12}$

A metodologia da consulta de enfermagem permite programar um plano de cuidados baseado nas características socioeconômicas, culturais e cognitivas individuais, levando em consideração a experiência e o conhecimento do paciente, família, grupo ou comunidade e realizar encaminhamentos a outras especialidades como: nutrição, odontologia, ginecologia e assistência social. ${ }^{12,13}$

A consulta de enfermagem pré-transplante renal visa identificar, qualificar e quantificar eventuais fatores que possam contraindicar a realização do transplante, ou que forneçam riscos transoperatórios e/ou pós-operatórios para que possam ser previamente tratados ou minimizados, de forma a tornar o transplante factível e com menores ricos para morbidade e mortalidade. ${ }^{13}$

A atividade transplantadora, além de se constituir em uma especialidade médica de bases técnicas e científicas, passa a ser uma especialidade com alta responsabilidade social, fazendo da seleção de doadores e receptores uma área de grande importância para os que se dedicam a essa especialidade.

\section{CONSIDERAÇÕES FINAIS}

A Consulta de Enfermagem pré-transplante renal propicia ao paciente e familiares um espaço de escuta ativa, diálogo, esclarecimento de dúvidas e o conhecimento da equipe de educação e tratamento. Iniciase neste momento a educação sobre o transplante renal e verifica-se a motivação do paciente para o tratamento como uma perspectiva para melhorar a realidade atual. A consulta de enfermagem com base modelo informatizado e no referencial teórico utilizado facilita o trabalho, proporciona comunicação efetiva e rápida entre a equipe multidisciplinar, permite interface com especialidades e áreas de apoio. Os dados do paciente ficam disponíveis em prontuário online para assistência, ensino e pesquisa.

\footnotetext{
ABSTRACT

Nursing consultation is a resource used by nurses aiming the patient's care, the family, groups or communities as to priorize the independency and autonomy of the individual regarding educative and preventive aspects. The assistance planning, risk factors, and nursing diagnosis detection added to interventions are all included in order to achieve the results. The purpose of this work is to describe the experience on renal pre-transplantation nursing consultation as an educative activity for patients candidate to a waiting list. Computerized physical assessment and nursing evolution models are used, which are elaborated according to Weed (subjective, objective, analysis or interpretation and plan or behavior), along with Wanda Horta's theory, priorizing basic human needs. This system (referred to as "Aplicativos de Gestão Hospitalar (AGH)", has been elaborated by professionals according to their areas of activity, as well as by the HCPA system analysis team. The experience of the pre-transplantation nursing consultation according to this model and theory provided the nurses the necessary information as to the patient's ability to perform the self-care, as well as the possibility to develop an individual care planning, taking into account the patient, his family, group or community's experience and knowledge. The same experience allowed to refer to other medical specialties such as: nutrition, odontology, gynecology and social working. In short, the pre-transplantation consultation provides the patient and family members the possibility of having a meaningful support from the care team, once they will be able to clarify any doubts and to establish an important dialogue with the caretakers.
}

Keywords: Nursing; Kidney transplantation; Outpatient Clinics, Hospital. 


\section{REFERÊNCIAS}

1. Brasil. Lei n. 7.498/86. Dispõe sobre a regulamentação do exercício da Enfermagem e dá outras providências. Brasília (DF): Conselho Federal de Enfermagem; [2000] [acessado em: 2 out 2009]. Disponível em: URL: http://www.portalcofen.gov. $\mathrm{br} / 2007 /$ materias.asp?ArticleID $=22 \&$ sectionID $=35$.

2. Silveira TF, Portela VCC. Consulta de enfermagem. Porto Alegre: UFRGS, Escola de Enfermagem; 2001 [acessado em: 5 out 2009]. Disponível em: URL: http://www.ufrgs. br/eenf/enfermagem/disciplinas/enf01004/material/consulta de enfermagem.pdf.

3. Silva MDG. A consulta de enfermagem no contexto da comunicação interpessoal: a percepção do cliente. Rev. Lat Am. Enfermagem. 1998;6(1):27-31.

4. Tasca AM, Santos BRL, Paskulin LMG, Záchia S. Cuidados ambulatoriais: consulta de enfermagem e grupos. Rio de Janeiro: EPUB; 2006.

5. Trezza MCSF, Santos RMD, Santos JMD. Trabalhando educação popular em saúde com a arte construída no cotidiano ad enfermagem: um relato de experiência. Texto Contexto Enferm. 2007;16(2):326-34.

6. Rios CTF, Vieira NFC. Ações educativas no pré-natal: reflexão sobre a consulta de enfermagem como um espaço para educação em saúde. Ciênc Saúde Colet 2007;12(2):477-86.
7. Fornazier ML, Siqueira MMD. Consulta de enfermagem a pacientes alcoolistas em um programa de assistência ao alcoolismo. J Bras Psiquiatr. 2006;55(4):280-7.

8. Weed LL. Medical records that guide and teach. N Engl J Med. 1968;278-593.

9. Horta WA. Processo de enfermagem. 14a. reimpr. 2001. São Paulo: EPU; 1979.

10. Rosenberger J, Geckova AM, van Dijk JP, Nagyova I, Roland R, van den Heuvel WJ, et al. Prevalence and characteristics of noncompliant behaviors and its risk factors in kidney transplant recipients. Transplant Int. 2005 Sept;18(9):1072-8.

11. Alvim NAT, Ferreira MDA. Perspectiva problematizadora da educação popular em saúde e a enfermagem. Texto Contexto Enferm. 2007;16(2):315-9.

12. Castro MCM, Aoki MVS, Domingos ES, Coutinho RCS, Silva CF, Couto JL, et al. Determinantes da não-adesão medicamentosa nos pacientes em hemodiálise. J Bras Nefrol. abr/jun 2009;31(2):89-95

13. Silva DS, Livramento ML, Pereira LM, David Neto E. Adesão ao tratamento imunossupressor no transplante renal. J Bras Nefrol. 2009;31(2):139-46.

14. Noronha IL, Manfro RC, Silva Filho APE. Manual de transplante renal. São Paulo: Manole; 2007. 